Recebido em 06/2019. Aceito para publicação em 03/2020.

\title{
EFEITO DA TERAPIA ROBÓTICA NO MEMBRO SUPERIOR PARÉTICO DE PACIENTES COM AVE
}

\section{EFFECT OF ROBOTIC THERAPY ON THE PARETIC UPPER LIMB OF STROKE PATIENTS}

\author{
Gabrielle Fernandes Neves ${ }^{1}$ \\ Diane Cristina Aleixo ${ }^{2}$ \\ Izabela Lopes Mendes ${ }^{3}$ \\ Mário Oliveira Lima ${ }^{4}$ \\ Sérgio Takeshi Tatsukawa de Freitas ${ }^{5}$ \\ Marcele Florêncio das Neves ${ }^{6}$
}

Resumo: Este artigo teve como objetivo analisar a resposta do músculo espástico do membro superior de indivíduos hemiparéticos, frente ao desempenho muscular e amplitude de movimento, após tratamento com terapia robótica. Como metodologia, a pesquisa serviu-se de uma amostra que consistiu em 12 voluntários sadios (Grupo Controle) e 15 voluntários pós-AVE (Grupo de Intervenção). Ambos grupos passaram por um protocolo de avaliação que consistiu em coleta de dados baseada na avaliação de fadiga e força muscular periférica: coleta de sangue para mensuração basal do lactato sanguíneo; avaliação de torque (Dinamômetro Isocinético); coleta de sangue para mensuração final do lactato sanguíneo; e avaliação funcional dos movimentos de flexão e extensão de cotovelo com auxílio da câmera Cinemática. O Grupo de Intervenção realizou 10 sessões de terapia robótica no exoesqueleto ArmeoSpring®, após o tratamento foi realizada a reavaliação. Após o tratamento, o Grupo de Intervenção apresentou aumento da amplitude de movimento, aumento do torque muscular e diminuição da concentração de lactato após uma contração voluntária máxima. Concluiu-se assim que a terapia robótica foi um recurso seguro e eficaz para melhora do desempenho do membro parético dos indivíduos que participaram deste estudo.

Palavras-chave: Terapia robótica; AVE; amplitude de movimento; torque; fadiga muscular.

Abstract: Objective: This article aimed to analyze the response of the spastic muscle of the upper limbs of hemiparetic individuals, in relation to muscle performance and range of motion, after treatment with robotic therapy. Methodology: the sample consisted of 12 healthy volunteers (Control Group) and 15 post-stroke volunteers (Intervention Group). Both groups underwent an evaluation protocol that consisted in data collection based on the evaluation of fatigue and peripheral muscle strength: blood collection for the baseline measurement of blood lactate; torque measurement (Isokinetic Dynamometer); blood collection for the final measurement of blood lactate; and functional evaluation of the elbow flexion and extension movements with the aid of the kinematic camera. The Intervention Group performed 10 sessions of robotic therapy on the ArmeoSpring $\AA^{8}$ exoskeleton, and after the treatment, the reevaluation was performed. Results: after the treatment, the Intervention Group presented increased range of motion, increased muscle torque and decreased lactate concentration after a maximal voluntary contraction. Conclusion: robotic therapy was a safe and effective tool to improve the performance of the paretic limb of the individuals who participated in this study.

Keywords: Robotic therapy; stroke; range of motion; torque; muscle fatigue.

\footnotetext{
${ }^{1}$ Fisioterapeuta, Universidade do Vale do Paraíba - Univap, Brasil. E-mail: gabrielle.neves@hotmail.com.

${ }^{2}$ Fisioterapeuta, Universidade do Vale do Paraíba - Univap, Brasil. E-mail: diane1994sjc@gmail.com.

${ }^{3}$ Docente do Curso de Fisioterapia, Universidade do Vale do Paraíba - Univap, Brasil. E-mail: izabela@univap.br.

${ }^{4}$ Docente do Curso de Fisioterapia, Universidade do Vale do Paraíba - Univap, Brasil. E-mail: mol@univap.br.

${ }^{5}$ Docente do Curso de Fisioterapia, Universidade do Vale do Paraíba - Univap, Brasil. E-mail: takeshi@univap.br.

${ }^{6}$ Docente do Curso de Fisioterapia, Universidade do Vale do Paraíba - Univap, Brasil. E-mail: mneves@univap.br.
} 


\section{INTRODUÇÂO}

O Acidente Vascular Encefálico (AVE) é definido pela Organização Mundial da Saúde (OMS) como desenvolvimento rápido de sinais clínicos de distúrbio focal (ou global) da função cerebral, com sintomas que duram 24 horas ou mais, causado por isquemia cerebrovascular ou hemorragia e que podem levar o paciente a óbito (BERTANI et al., 2017; TANAKA et al., 2019).

A falta súbita de suprimento sanguíneo gera um déficit na oxigenação encefálica, com consequente morte neuronal, gerando complicações nas funções cognitivas, motora e sensorial que impedem o indivíduo de realizar atividades da vida diária de forma independente (AMARAL et al., 2017). Dentre as principais complicações pós-AVE está a espasticidade, definida como um distúrbio sensório-motor causado por uma lesão do motoneurônio superior e caracterizada por uma ativação involuntária intermitente ou sustentada dos músculos antigravitacionais (REKAND et al., 2019).

A espasticidade pode causar atrofia e encurtamento muscular e tendíneo, resultando em diminuição da força muscular e da amplitude de movimento (SUN et al., 2019). O músculo esquelético é composto por diferentes tipos de fibras, porém há predomínio de um tipo específico. As fibras musculares do tipo 1 possuem alta concentração de enzimas de metabolismo aeróbio, mioglobina, e maior densidade de vascularização, sendo mais resistentes à fadiga, são de contração lenta e metabolismo oxidativo. As fibras musculares do tipo 2 possuem baixa resistência à fadiga, são de contração rápida e metabolismo glicolítico (MINAMOTO, 2005). O músculo espástico quando comparado ao músculo não espástico possui uma proporção menor de fibras do tipo 1 e maior de fibras do tipo 2, que são mais rápidas e fadigáveis, além de apresentar diminuição no tamanho das fibras musculares e redução da atividade enzimática oxidativa. Estas alterações comprometem o desempenho muscular tornando o músculo espástico mais propenso à fadiga (SEVERINSEN et al., 2016).

A espasticidade no membro superior é uma condição comum em indivíduos acometidos por AVE, sendo esta a disfunção que afeta a independência, qualidade de vida e humor de cerca de $80 \%$ dos sobreviventes pós AVE (CALABRÒ et al., 2017; MCLAREN et al., 2019). A terapia robótica surge com o propósito de promover aprendizagem motora do membro superior parético utilizando cenários virtuais com avatares que apresentam os movimentos do membro ou objetos com os quais o indivíduo deve interagir (COLOMER et al., 2013), e dispõe de dispositivos que podem mover de modo assistido o membro acometido pelo AVE, oferecendo assistência ou resistência ao movimento (POLLOCK et al., 2014).

Considerando a terapia robótica uma promessa para reabilitação do membro superior, este artigo teve como objetivo analisar a resposta do músculo espástico do membro superior de indivíduos hemiparéticos, frente ao desempenho muscular e amplitude de movimento, após tratamento com terapia robótica. 


\section{METODOLOGIA}

Trata-se de um estudo experimental de caráter analítico transversal, projetado como um ensaio clínico controlado duplo-cego. Este estudo foi aprovado pelo Comitê de Ética em Pesquisa (CEP) da Universidade do Vale do Paraíba (UNIVAP) sob protocolo CAAE: 40764115.5.0000.5503. Todos os participantes foram informados sobre os procedimentos do estudo e assinaram um formulário de consentimento.

A amostra consistiu em 12 voluntários sadios (5 do gênero masculino e 7 do gênero feminino) com idade média de 58,6 \pm 9 anos e 15 voluntários pós-AVE (9 do gênero masculino e 6 do feminino) com diagnóstico de hemiparesia espástica com predomínio braquial, com média de idade de 59,9 \pm 12 anos. O estudo teve início entre 11 meses e 48 meses após a ocorrência do AVE.

Os voluntários sadios foram inseridos no Grupo Controle e os voluntários pós-AVE foram inseridos no Grupo de Intervenção, e foram determinados os protocolos de avaliação para ambos os grupos e o tratamento para o Grupo de Intervenção conforme Figura 1.

Após a seleção e divisão dos voluntários nos grupos supracitados, foi realizada a avaliação manual da força muscular e avaliação do tônus muscular segundo a Escala de Ashworth Modificada, seguidas pela coleta de dados baseada na avaliação de fadiga e força muscular periférica: coleta de sangue para mensuração basal do lactato sanguíneo; avaliação do torque durante contração voluntária máxima (CVM) de bíceps braquial no Dinamômetro Isocinético; coleta de sangue para mensuração final do lactato sanguíneo; e avaliação funcional dos movimentos de flexão e extensão de cotovelo com auxílio da câmera Cinemática com a avaliação da amplitude de movimento. Os voluntários sadios do Grupo Sadio realizaram todo o processo de avaliação ao qual foram submetidos os pacientes pós-AVE, com exceção da avaliação manual de força muscular e Escala de Ashworth Modificada. 
Figura 1 - Fluxograma indicando a divisão dos grupos e a sequência dos protocolos de avaliação e tratamento.
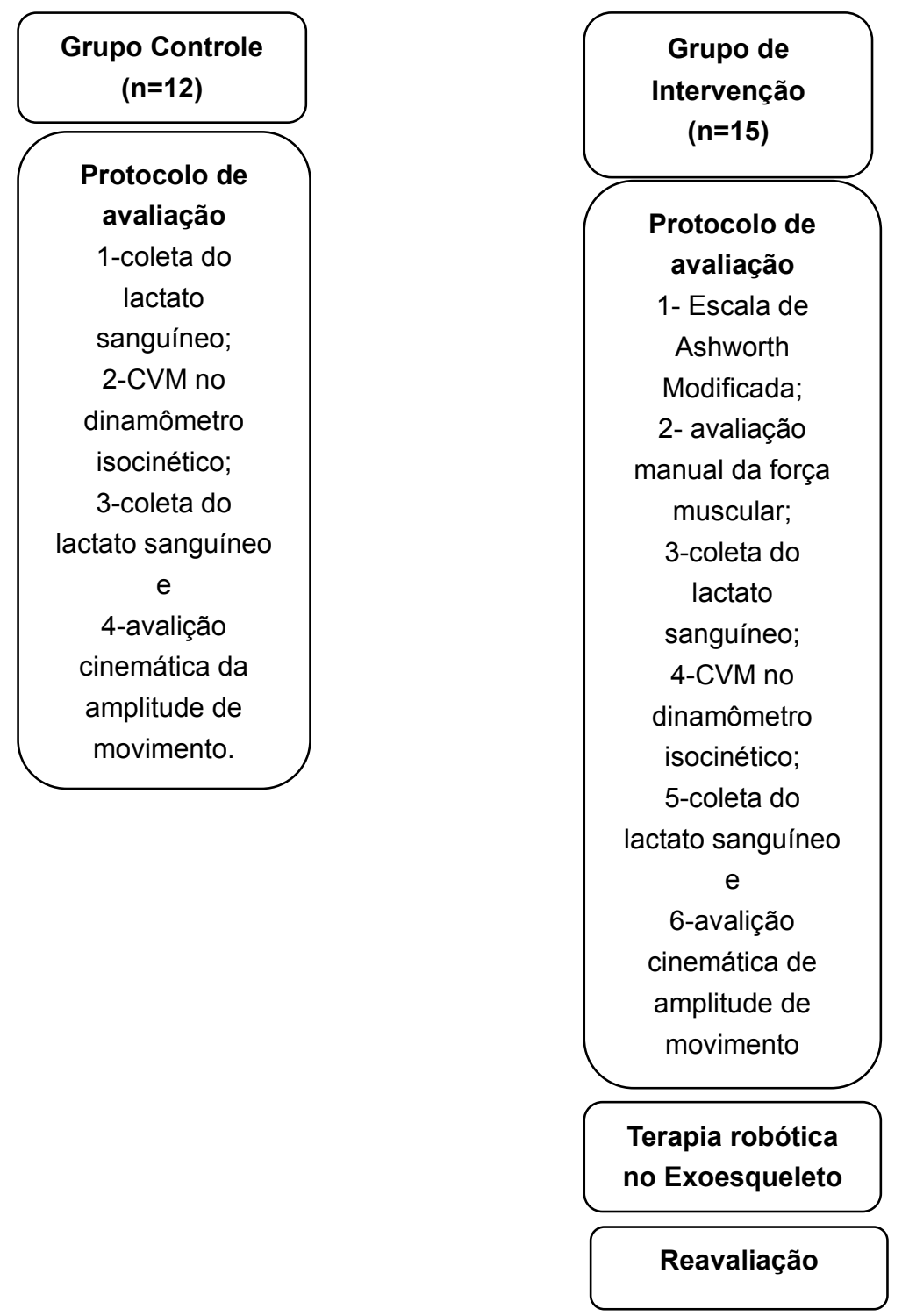

Fonte: Os autores.

\subsection{Lactímetro}

Para a coleta dos níveis de lactato sanguíneo, foi utilizado o Lactímetro Accutrend $₫$ PLUS da Roche, em dois momentos: primeiramente, na chegada e acomodação do voluntário, no local da coleta (medida basal), e posteriormente a realização da contração muscular máxima no dinamômetro isocinético (medida final). $A$ coleta do sangue venoso foi realizada mediante a perfuração da polpa do dedo, preferencialmente das falanges distais, com auxílio de uma lanceta descartável. A gotícula de sangue foi colocada sobre a fita de dosagem lactato BM_lactate (Roche Accusport- Tiras'25) próprias para o equipamento e assim analisadas pelo lactímetro portátil (Accutrend® PLUS da Roche). 
Ao término da avaliação, os valores da concentração de lactato sanguíneo foram anotados e comparados, ou seja, valor basal com o valor pós-exercício isométrico para a confirmação do estado de fadiga muscular.

\subsection{Dinamometria Isocinética}

A mensuração do torque muscular foi realizada a partir da contração isométrica do músculo bíceps braquial, para a qual foi utilizado dinamômetro isocinético da marca Biodex System ${ }^{\circledR}$ 3. O voluntário foi posicionado adequadamente em sedestação na cadeira do equipamento, com os pés sobre um suporte e estabilizados com cintas de estabilização, posicionadas nos membros inferiores e outras duas cruzadas sobre 0 tronco. O braço de alavanca foi conectado a um acessório de punho para posicionar a mão, estabilizada com a utilização de atadura. O cotovelo em $90^{a}$ de flexão ficou apoiado sobre uma almofada de apoio e o eixo do dinamômetro posicionado de modo que coincida com o eixo do movimento de flexão e extensão da articulação do cotovelo. $O$ dinamômetro foi acionado e o voluntário foi instruído a realizar a contração isométrica voluntária máxima de flexor de cotovelo (bíceps braquial) durante 50 segundos. Concomitante a este, foi solicitado o máximo de empenho do voluntário, mediante estímulo de comandos verbais para a realização de força máxima, o que permitirá a quantificação do torque muscular.

Para a realização do teste isométrico, foi criado um protocolo com os seguintes dados:

- Modo: Unilateral Isométrico;

- Articulação: Cotovelo;

- Padrão: Extensão/ Flexão;

- Direção da Contração: Toward;

- Posição: 1;

- Repetições: 1;

- Ângulo: $90^{\circ}$;

- Tempo do Teste: 50 segundos;

- Tempo de Relaxamento: 0;

- Referência Anatômica: 90․

Após coleta, os dados foram exportados para um sistema de banco de dados e assim analisados pelo software OriginLab®, utilizando filtro band pass de $4^{\underline{a}}$ ordem Butterworth, ajustado para frequência de corte de $20 \mathrm{~Hz}$ a $400 \mathrm{~Hz}$ para eliminar ruído residual. Em seguida foi feito o cálculo do Coeficiente linear, pelo qual obteve-se o valor médio dos picos de torque. 


\subsection{Cinemática}

O local da realização dos testes foi inicialmente demarcado com fita adesiva, medindo 2,70m². Em seguida, foi marcado o posicionamento exato da cadeira e da mesa, utilizadas no teste. A mesa foi posicionada na região central do espaço demarcado para a coleta, a uma distância de $60 \mathrm{~cm}$ da marcação onde a cadeira ficará posicionada, essas medidas foram respeitadas para todos os pacientes a fim de padronizar a coleta em relação às câmeras e sua calibração.

As duas câmeras de vídeo da marca Optitrack, modelo Flex: V100 R2, foram apoiadas nos tripés, e posicionadas lateralmente a área de coleta, e, para a reconstrução tridimensional, cada marcador foi visível por ambas simultaneamente. Os marcadores foram posicionados no ombro (tubérculo maior do úmero), no cotovelo (epicôndilo lateral) e no punho (processo estiloide da ulna).

Os voluntários foram posicionados em sedestação diante da caixa interativa, com $90^{\circ}$ de flexão de quadril, joelho e tornozelo com pés apoiados no chão, tronco estabilizado no encosto da cadeira usando uma faixa na altura do peito para minimizar movimentos compensatórios, a distância do membro parético até a caixa foi determinada pelo comprimento funcional do braço (da linha axilar anterior até a prega do punho, quando o indivíduo eleva seu braço mais próximo a $90^{\circ}$ de flexão possível no plano sagital). Após o posicionamento a caixa foi acionada no modo "2 botões" e o voluntário foi instruído a alcançar e pressionar com o membro superior parético dois botões específicos que acenderão, em duas diferentes alturas (botão número 6 e botão número 16, localizados na segunda linha/segunda coluna, e quarta linha/quarta coluna respectivamente), no menor tempo possível, respeitando a harmonia do movimento, totalizando 10 movimentos de alcance, 5 para cada altura.

\subsection{Tratamento}

\subsubsection{Aquecimento}

Foi realizado um programa de exercícios baseado em:

- Repouso de 10 minutos após a chegada ao local;

- Verificação dos sinais vitais (frequência cardíaca, frequência respiratória, pressão arterial);

- Mobilização articular passiva dos componentes osteomioarticulares do membro superior parético (ombro, cotovelo e punho), 3 séries de 10 repetições dos movimentos angulares de cada articulação: circundação do ombro, que combina sequencialmente os movimentos de flexão, abdução, extensão e adução; flexão, extensão, supinação e pronação de cotovelo; circundação do punho, que acompanha os movimentos sucessivos de flexão, abdução, extensão e adução; 
- Alongamento passivo de bíceps braquial: extensão do cotovelo com o antebraço em pronação, por duas repetições de 30 segundos, com intervalo de 30 segundos de repouso entre uma e outra;

- Alongamento passivo de tríceps braquial: flexão de cotovelo, com antebraço em pronação e o ombro semi-flexionado, por duas repetições de 30 segundos, com intervalo de 30 segundos de repouso, durante os quais foram realizados os alongamentos passivos de bíceps braquial.

\subsubsection{Terapia Robótica - Exoesqueleto (Armeo ${ }^{\circledR}$ Spring)}

Os pacientes do Grupo de Intervenção foram submetidos a 10 sessões de terapia robótica com exoesqueleto Armeo®Spring (HOCOMA), uma réplica comercial do TWREX, utilizada para o treinamento do membro superior afetado. $O$ braço do equipamento foi ajustado ao tamanho do paciente e permitiu um suporte contra a ação da gravidade, sustentando $45^{\circ}$ de flexão de ombro, o que facilitou a movimentação destes pacientes, utilizando a função residual do membro superior parético.

Figura 2 - Exoesqueleto Armeo ${ }^{\circledR}$ Spring (HOCOMA).

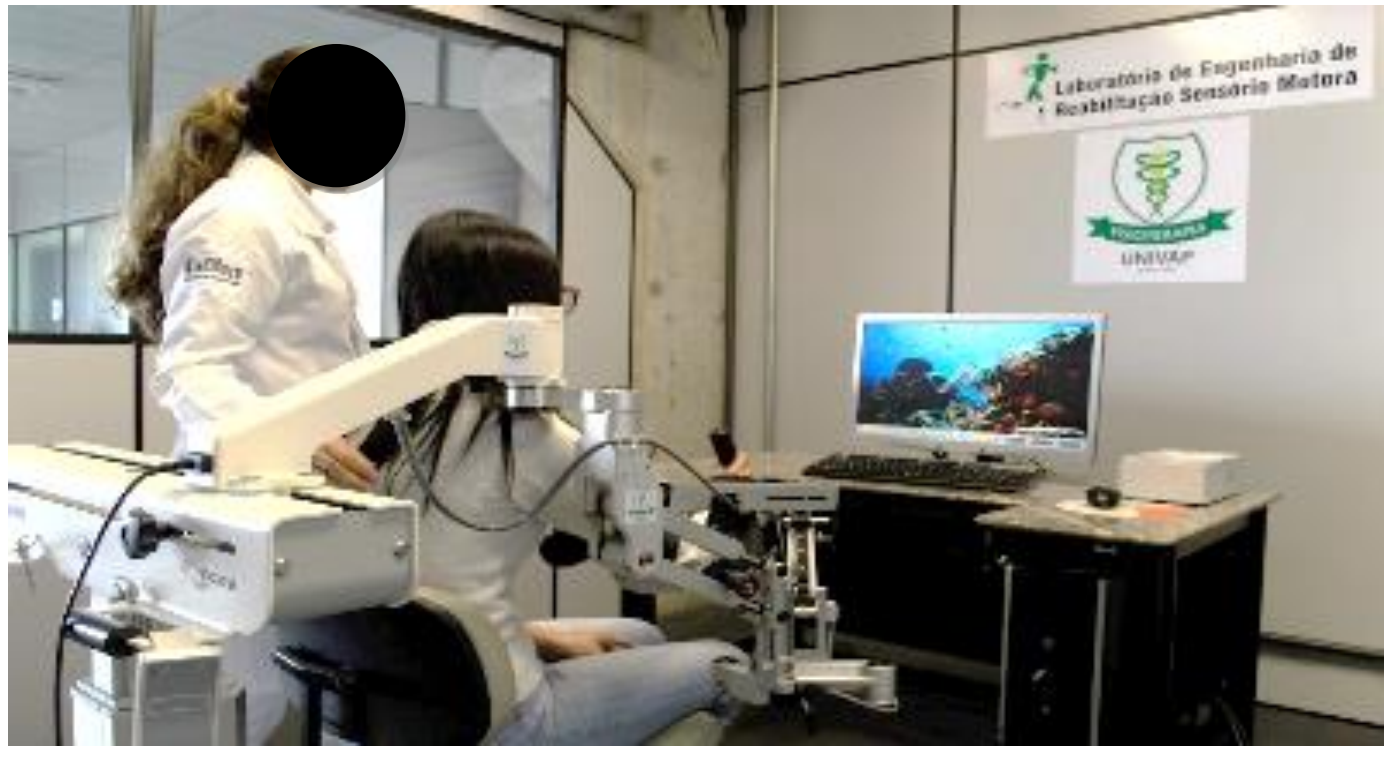

Fonte: Os autores.

As etapas de treinamento foram gradualmente ajustadas a partir da primeira sessão, a cada semana. A terapia foi aplicada 3 vezes por semana totalizando 10 sessões. Cada sessão teve duração de 30 minutos e consistiu de um plano terapêutico dividido em 3 exercícios funcionais:

1. Movimento grosso (Limpar janela) - 10 minutos. $O$ exercício consistiu na realização de um movimento grosseiro de punho, mão, cotovelo e ombro, no plano frontal, onde o paciente interage com a interface limpando a janela com uma esponja. Há 4 níveis de dificuldade, nos quais quando aumenta o nível a esponja fica menor, sendo necessário um movimento mais preciso para cobrir a superfície e, no modo 
de preensão automática desligada, o punho tem que ser continuamente pressionado para ativar a esponja;

Figura 3 - Interface do exercício funcional de limpar a janela no exoesqueleto.

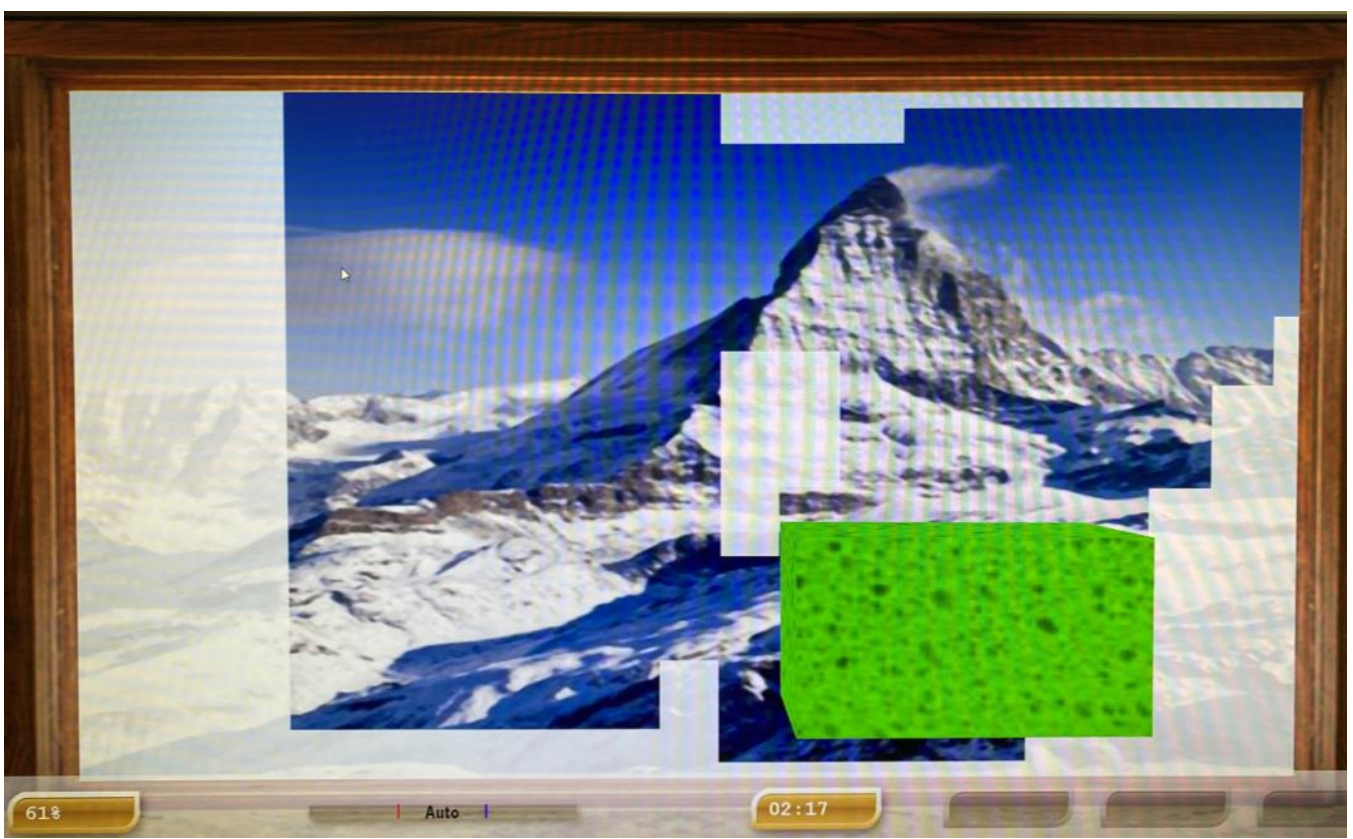

Fonte: Armeo ${ }^{\circledR}$ Spring (HOCOMA).

2. Movimento preciso (Pesca) - 10 minutos. O exercício consistiu na movimentação, no plano frontal, de punho, mão, cotovelo e ombro, onde o paciente deve interagir com a interface pescando os peixes com uma rede. Há 4 níveis de dificuldade, nos quais com o aumento do nível, os peixes ficam cada vez menores, requisitando do paciente movimentos mais precisos;

Figura 4 - Interface do exercício funcional de pesca no exoesqueleto.

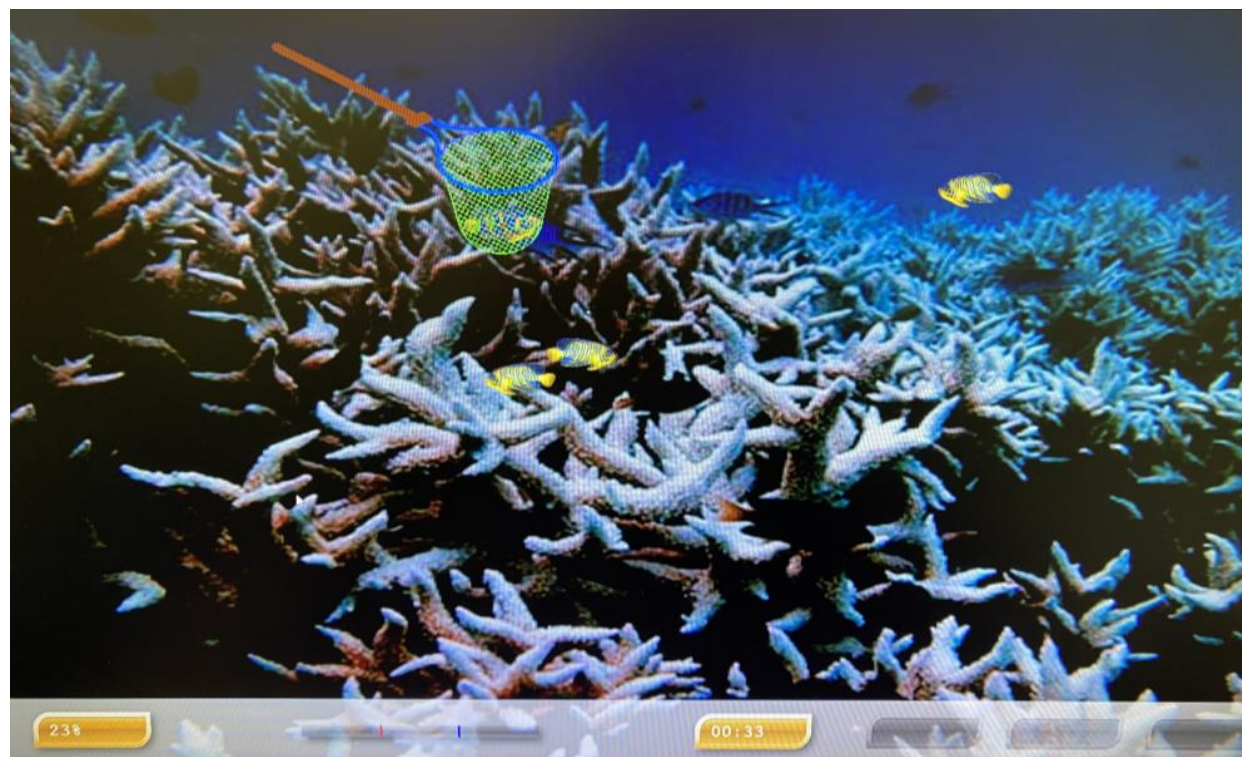

Fonte: Armeo ${ }^{\circledR}$ Spring (HOCOMA). 
3. Movimento fino - 10 minutos. O exercício consistiu na realização de movimentos da mão no plano frontal, onde o paciente precisa sustentar o movimento durante 3 segundos para apanhar as moscas com o mata-moscas, necessitando precisão e coordenação. Há 4 níveis de dificuldade, onde há o aumento da área de trabalho e do número de objetos.

Figura 5 - Interface do exercício funcional de apanhar a mosca no exoesqueleto.

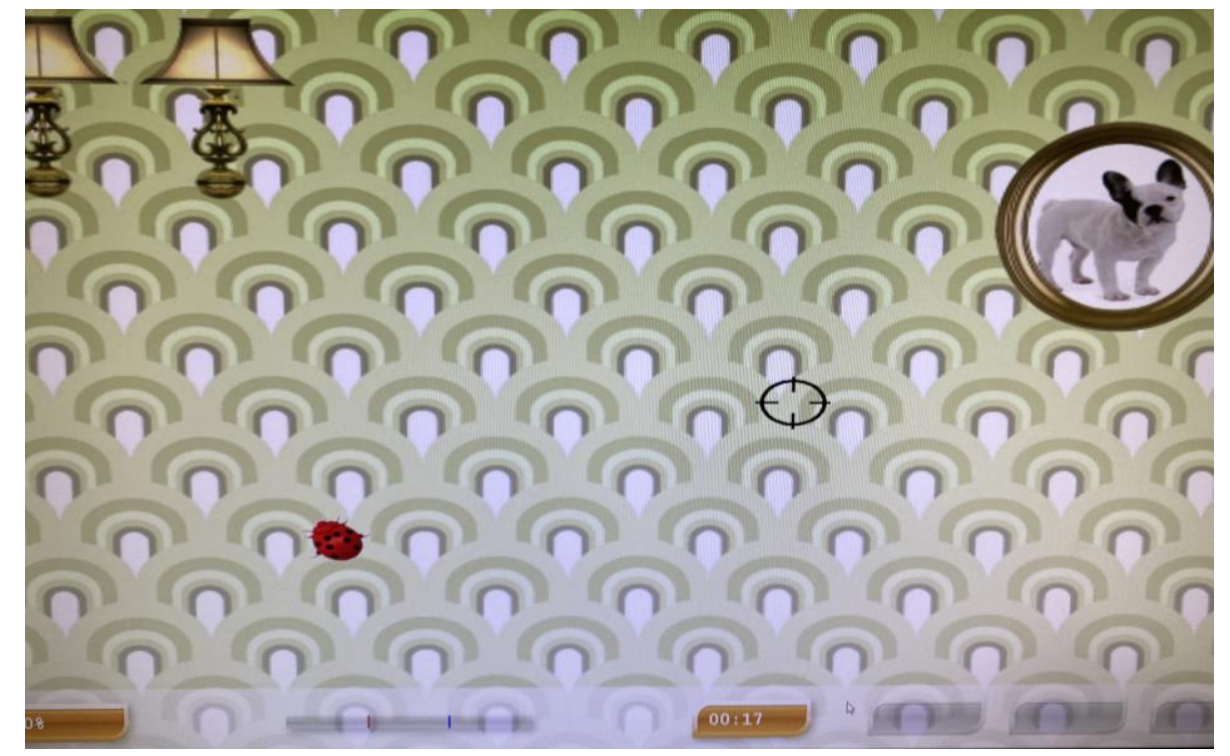

Fonte: Armeo ${ }^{\circledR}$ Spring (HOCOMA).

\subsection{Análise estatística}

Realizou-se a análise estatística dos dados no programa BioEstat@ versão 5.3. Para análise descritiva utilizou-se a tendência central e o desvio padrão $( \pm D P)$, com valores máximos e mínimos. Os dados passaram pelo Teste de Normalidade KS (Kolmogorov-Smirnov). Utilizou-se o teste estatístico Two Way Anova para amostras relacionadas nas análises intragrupo e intergrupo. O alfa foi ajustado para um nível de significância de $p=0,05$.

\section{RESULTADOS}

Os valores de lactato sanguíneo foram apresentados na Tabela 1; o torque do músculo bíceps braquial está presente na Tabela 2; e os dados de amplitude de movimento foram apresentados na Tabela 3.

Tabela 1 - Valores da Concentração de Lactato antes e depois da realização da CVM. Valores representados em média e um erro padrão

\begin{tabular}{|c|c|c|c|c|c|c|}
\hline \multicolumn{7}{|c|}{ Lactato Sanguíneo (mmol/L) } \\
\hline \multirow{2}{*}{ Grupo } & \multicolumn{2}{|c|}{ Pré-tratamento } & \multicolumn{2}{|c|}{ Pós tratamento } & & \\
\hline & Antes & Depois & Antes & Depois & $\begin{array}{c}\text { Valor de p } \\
\text { (Intragrupo) }\end{array}$ & $\begin{array}{c}\text { Valor de p } \\
\text { (Intergrupo) }\end{array}$ \\
\hline
\end{tabular}


Tabela 1 - Valores da Concentração de Lactato antes e depois da realização da CVM. Valores representados em média e um erro padrão (conclusão).

\begin{tabular}{|c|c|c|c|c|c|c|}
\hline Controle & $2,7 \pm 0,6$ & $4,4 \pm 0,5$ & - & - & & \\
\hline \multirow{4}{*}{ Intervenção } & \multirow{4}{*}{$4,1 \pm 1,3$} & \multirow{4}{*}{$5,6 \pm 1,3$} & \multirow{4}{*}{$3,3 \pm 1,1$} & \multirow{4}{*}{$4,9 \pm 1,5$} & $\begin{array}{l}\text { (Pré-Pré) } \\
\mathrm{P}=0,0779\end{array}$ & $\begin{array}{c}\text { (Controle } x \\
\text { Pré antes) } \\
0,0010\end{array}$ \\
\hline & & & & & \multirow{3}{*}{$\begin{array}{l}\text { (Pós-Pós) } \\
P=0,3505\end{array}$} & $\begin{array}{c}\text { (Controle x } \\
\text { Pré depois) } \\
0,0838\end{array}$ \\
\hline & & & & & & $\begin{array}{c}\text { (Controle x } \\
\text { Pós antes) } \\
0,0036\end{array}$ \\
\hline & & & & & & $\begin{array}{c}\text { (Controle x } \\
\text { Pós depois) } \\
0,0707\end{array}$ \\
\hline
\end{tabular}

Fonte: Autores.

Os resultados referentes à concentração de lactato sanguíneo mostram que os indivíduos do Grupo Controle apresentam aumento do lactato após CVM menor que os indivíduos do Grupo de Intervenção, exceto se comparada a concentração de lactato depois da CVM do Grupo de Intervenção e a concentração de lactato do Grupo de Intervenção após CVM depois do protocolo de tratamento. Em relação à concentração de lactato sanguíneo depois do protocolo tratamento do Grupo de Intervenção, é possível observar que houve uma diminuição do lactato tanto antes quanto após a CVM, mostrando melhora do desempenho muscular em relação à fadiga após terapia robótica.

Tabela 2 - Torque em Newton-metro (N.m) do músculo bíceps braquial, antes e após protocolo de tratamento, expresso como média \pm DP durante a CVM realizada no Dinamômetro Isocinético. (continuação)

\begin{tabular}{|c|c|c|c|c|}
\hline & \multicolumn{3}{|c|}{ Torque (N.m) } \\
\hline Grupo & Antes & Depois & $\begin{array}{c}\text { Valor de } p \\
\text { (Intragrupo) }\end{array}$ & $\begin{array}{c}\text { Valor de } p \\
\text { (Intergrupo) }\end{array}$ \\
\hline Controle & $48,9 \pm 14$ & Não aplicado & & \\
\hline Intervenção & $22,0 \pm 14$ & $24,0 \pm 14,6$ & P=0,7314 & $\begin{array}{c}\text { (Controle } x \\
\text { Antes) }\end{array}$ \\
\hline
\end{tabular}


Tabela 2 - Torque em Newton-metro (N.m) do músculo bíceps braquial, antes e após protocolo de tratamento, expresso como média \pm DP durante a CVM realizada no Dinamômetro Isocinético. (continuação) (Controle $\mathrm{x}$ Depois) $<0,0001$

\section{Fonte: Autores.}

É possível observar que o torque do Grupo Controle foi significativamente maior que o do Grupo de Intervenção antes e depois do protocolo de tratamento. Após o tratamento houve um aumento significativo do torque do Grupo de Intervenção.

Tabela 3 - Amplitude de movimento (graus) antes e depois do protocolo de tratamento expresso como média \pm DP.

\begin{tabular}{|c|c|c|c|c|}
\hline \multirow[b]{2}{*}{ Grupo } & \multicolumn{4}{|c|}{ Amplitude de Movimento (graus) } \\
\hline & Antes & Depois & $\begin{array}{l}\text { Valor de } \mathrm{p} \\
\text { (Intragrupo) }\end{array}$ & Valor de p (Intergrupo) \\
\hline Controle & $109,4 \pm 9,6$ & $\begin{array}{c}\text { Não } \\
\text { aplicado }\end{array}$ & & Não aplicado \\
\hline \multirow{3}{*}{ Intervenção } & \multirow{3}{*}{$67,8 \pm 29,4$} & \multirow{3}{*}{$71,6 \pm 26,4$} & \multirow{3}{*}{$p=0.7014$} & $\begin{array}{c}\text { (Controle } x \text { Intervenção } \\
\text { antes) } p=0,0010\end{array}$ \\
\hline & & & & \\
\hline & & & & $\begin{array}{c}\text { (Controle } \mathrm{x} \text { Intervenção } \\
\text { depois) } \\
\mathrm{p}<0.0001\end{array}$ \\
\hline
\end{tabular}

Fonte: Autores.

A amplitude de movimento do Grupo Controle foi significativamente maior quando comparada à do Grupo de Intervenção antes e após o protocolo de tratamento. Entretanto, comparando a amplitude de movimento do Grupo de Intervenção, antes e depois do protocolo de tratamento, observa-se um aumento significativo da amplitude de movimento.

\section{DISCUSSÃO}

O objetivo do presente estudo foi analisar a resposta do músculo espástico do membro superior de indivíduos hemiparéticos, frente ao desempenho muscular e amplitude de movimento, após tratamento com terapia robótica. Duret, Grosmaire e Krebs (2019) concluíram em seu estudo que a terapia robótica oferece intensidade, interatividade e adaptabilidade ao desempenho e às necessidades dos indivíduos pósAVE, focando em melhorar o membro comprometido de acordo com os conceitos da 
neuroplasticidade.

Os resultados do estudo mostraram que a amplitude de movimento (ADM) observada na análise cinemática dos indivíduos pós-AVE é significativamente inferior quando comparada aos indivíduos sadios mesmo após o protocolo de tratamento com a terapia robótica. Os autores Grimm, Naros e Garabaghi (2016), também apresentaram dados mostrando o déficit de ADM de pacientes acometidos pelo AVE quando comparados a indivíduos sadios, mesmo após o tratamento de 20 sessões com o exoesqueleto. Entretanto, para o estudo houve aumento significativo da amplitude de movimento dos indivíduos pós-AVE após o tratamento proposto, resultado que também está de acordo com Grimm, Naros e Garabaghi (2016), que observaram reaprendizagem motora, melhora na amplitude de movimento e evolução na qualidade do movimento em indivíduos pós-AVE. Um estudo realizado por Adomavičienè et al. (2019) também mostrou melhora na amplitude de movimento do ombro e cotovelo do membro parético de indivíduos pós-AVE depois de tratamento com terapia robótica.

A realização de um treinamento intensivo da função motora, como a terapia robótica, induz o sistema nervoso central a explorar áreas motoras não afetadas pelo AVE gradativamente, o que consequentemente irá minimizar as sequelas da espasticidade, como a rigidez muscular, melhorando a amplitude de movimento e habilidade funcional do indivíduo (NEVES, 2017).

Após o tratamento com terapia robótica o Grupo de Intervenção apresentou melhora significativa do torque do músculo bíceps braquial. Colomer et al. (2013) realizaram um estudo com o objetivo de avaliar a eficácia de um programa de reabilitação com o Armeo®Spring em pacientes com hemiparesia crônica após AVE com escalas dirigidas para os domínios de estrutura (Escala de Ashworth Modificada), função (Motricity Index e Fugl Meyer Assessment Scale) e atividade (Motor Assessment Scale Manual Function Test e Wolf Motor Function) da CIF (Classificação Internacional de Funcionalidade, Incapacidade e Saúde). Este estudo mostrou melhora significativa nos domínios da função e atividade, com exceção do Motricity Index, de pacientes pós-AVE após tratamento com terapia robótica, concordando com os achados deste estudo.

Por meio da prática repetitiva e treinamento com dispositivo robótico é possível ativar neurônios corticais adjacentes aos que foram lesionados, gerando plasticidade neuronal e, consequentemente, adequação do tônus muscular e melhora da força muscular (BORGES et al., 2014).

O protocolo de terapia robótica proporcionou, no presente estudo, diminuição da concentração de lactato antes e depois de uma contração voluntária máxima, indicando melhora no desempenho muscular. Com a prática de exercícios regulares, de intensidade aeróbica capaz de gerar ácido lático, o tempo de ressíntese das moléculas de ATP pode ser otimizado e o tempo de oxidação de ácido lático minimizado, proporcionando resistência física e metabólica a exercícios de alta intensidade com consequente redução da fadiga (LIMA; CABRAL; CAMPOS, 2018). 


\section{CONCLUSÃO}

Este artigo teve como objetivo analisar a resposta do músculo espástico do membro superior de indivíduos hemiparéticos, frente ao desempenho muscular e amplitude de movimento, após tratamento com terapia robótica. Os resultados foram positivos em relação ao aumento de força, amplitude de movimento e diminuição da fadiga, sugerindo que a terapia robótica foi um recurso seguro e eficaz para melhora do desempenho do membro parético dos indivíduos que participaram deste estudo.

\section{AGRADECIMENTO}

O presente trabalho teve apoio fornecido pela FINEP/MCTIC - Convênio no 01.13.0275.00 e Coordenação de Aperfeiçoamento Pessoal de Nível Superior (CAPES - PE 030/2008).

\section{REFERÊNCIAS}

ADOMAVIČIENĖ, A. et al. Influence of New Technologies on Post-Stroke Rehabilitation: A Comparison of Armeo Spring to the Kinect System. Medicina, v. 55, n. 98, 2019.

AMARAL, D. B. S. et al. Avaliação da funcionalidade do membro superior parético de pacientes com sequela de ave após protocolo terapia por contensão induzida. Revista Perspectivas Online: Biológicas \& Saúde, v. 7, n. 28, p. 81-87, 2017.

BERTANI, R. et al. Effects of robot-assisted upper limb rehabilitation in stroke patients: a systematic review with meta-analysis. Neurological Sciences, v. 38, n. 9, p.15611569, 2017.

BORGES, A. C. L. et al. Terapia de repetição utilizando dispositivo eletrônico com estímulo visual em hemiparéticos espásticos. In: CONGRESSO BRASILEIRO DE ENGENHARIA BIOMÉDICA, 14., 2014. Anais..., 2014, p. 573-576.

CALABRÒ, R. S. et al., Is two better than one? Muscle vibration plus robotic rehabilitation to improve upper limb spasticity and function: A pilot randomized controlled trial. PLoS ONE, v. 12, n. 10, 2017.

COLOMER, C. et al. Eficacia del sistema Armeo®Spring en la fase crónica del ictus. Estudio en hemiparesias leves-moderadas. Neurología, v. 28, n. 5, p. 261-267, 2013.

DURET, C.; GROSMAIRE A. C.; KREBS, H. I. Robot-Assisted Therapy in Upper Extremity Hemiparesis: Overview of an Evidence-Based Approach. Front. Neurol, v. 10, n. 412, 2019.

GRIMM, F.; NAROS, G.; GHARABAGHI, A. Compensation or restoration: closed-loop feedback of movement quality for assisted reach-to-grasp exercises with a multi-joint arm exoskeleton. Rev Neurocience, v. 10, p. 1-8, 2016.

LIMA, I. J. D.; CABRAL, T. B.; CAMPOS, C. C. C. Avaliação de índices fisiológicos em indivíduos não praticantes de exercícios físicos que utilizam a realidade virtual e aumentada: um estudo piloto. Revista Eletrônica Estácio Recife, v. 4, n. 2, 2018. 
MCLAREN, R. et al. The volume and timing of upper limb movement in acute stroke rehabilitation: still room for improvement. Disability and Rehabilitation, p. 1-6, 2019.

MINAMOTO, V. B. Classificação e adaptações das fibras musculares: uma revisão. Fisioterapia e Pesquisa, v. 12, n. 3, p. 50-55, 2005.

NEVES, M. F. Efeito da terapia a laser de baixa intensidade sobre a atividade do músculo espástico submetido à terapia robótica. 2017. $87 \mathrm{f}$. Tese (Doutorado em Engenharia Biomédica) - Universidade do Vale do Paraíba, São José dos Campos, 2017.

POLLOCK, A. et al. Interventions for improving upper limb function after stroke (Review). Cochrane Database of Systematic Reviews, v. 2014, n. 11, 2014.

REKAND, T. et al. Botulinum toxin treatment of spasticity targeted to muscle endplates: an international, randomised, evaluatorblinded study comparing two different botulinum toxin injection strategies for the treatment of upper limb spasticity. BMJ Open, v. 9, n. 5, 2019.

SEVERINSEN, K. et al. Skeletal muscle fiber characteristics and oxidative capacity in hemiparetic stroke survivors. Muscle \& Nerve, v. 53, n. 5, p. 748-754, 2016.

SUN, Li-Chun et al. Efficacy and Safety of Botulinum Toxin Type A for Limb Spasticity after Stroke: A Meta-Analysis of Randomized Controlled Trials. BioMed Research International, v. 2019, 2019.

TANAKA, H. et al. Spatiotemporal gait characteristic changes with gait training using the hybrid assistive limb for chronic stroke patients. Gait \& Posture, v. 71, p. 205-210. 2019. 